\title{
Um modelo para a produção de Objetos de Aprendizagem Acessíveis: Modelagem e Análise por Redes de Petri Coloridas
}

\author{
Corneli Gomes Furtado Júnior ${ }^{1,2,3}$, José Marques Soares ${ }^{2}$, Giovanni Cordeiro Barroso ${ }^{2}$, \\ Lidiane Castro $^{3}$, Maikon Igor da Silva Soares ${ }^{1,3}$, Cassandra Ribeiro Joye ${ }^{1,3}$, \\ Adriano Tavares de Freitas ${ }^{1,3}$, Éder Furtado Soares ${ }^{3}$ \\ ${ }^{1}$ Instituto Federal de Educação Ciência e Tecnologia do Ceará (IFCE) \\ Av. Treze de Maio, 2081 - Fortaleza - Ce - Brasil \\ ${ }^{2}$ Universidade Federal do Ceará - UFC \\ Departamento de Teleinformática - Deti - Centro de Tecnologia. \\ ${ }^{3}$ Laboratório de Educação a Distância para Pessoas com Deficiência (Le@ @) \\ Av. Santos Dumont, 2456 - Aldeota \\ cjuniordifce.edu.br, marques@ufc.br,gcb@fisica.ufc.br \\ \{lidcasto, maikonigor, cassandra, adrianoblue, ederfurtado\} @gmail.com
}

\begin{abstract}
In this work we present a model for the development of Learning $O b$ jects. The model was constructed and evaluated using Coloured Petri Nets, and the results presented in this article. The adoption of model allows the specification and analysis of the flow of production as well as sizing of team and identification of bottlenecks in production in general.
\end{abstract}

Resumo. Neste trabalho apresentamos um modelo para o desenvolvimento de Objetos de Aprendizagem Acessíveis. O modelo foi construído e avaliado por Redes de Petri Coloridas, e os resultados apresentados neste artigo. A adoção do modelo permite a especificação e análise do fluxo de atividades da produção dos conteúdos, bem como dimensionamento da equipe e identificação de gargalos na produção em geral.

\section{Introdução}

O Brasil possui 45 milhões de pessoas com deficiência (PCD) [IBGE 2014], uma parcela importante da população, que hoje possui poucos recursos tecnológicos a favor da formação profissional. Por outro lado, temos um mercado de trabalho promissor que, a cada dia, reforça o problema da falta de profissionais qualificados. É necessário, portanto, a implantação de soluções tecnológicas capazes de qualificar um grande número de PCDs, atendendo às necessidades de acessibilidade deste público.

O Laboratório de Educação a Distância para Pessoas com Deficiência (Le@d), criado a partir do investimento da empresa Dell Computadores, pesquisa e desenvolve tecnologias e conteúdos de Educação a Distância $(\mathrm{EaD})$ aplicados à formação de pessoas com deficiência física e pessoas com surdez. Todos os materiais e ferramentas construídos são validados por uma equipe de testadores formada por pessoas com essas deficiências. Dessa forma, os artefatos criados são aprimorados de acordo com a visão do usuário final, para que o aluno receba um material de qualidade e adaptado às suas necessidades de aprendizagem. 
Assim como no desenvolvimento de software, o desenvolvimento de conteúdos didáticos no formato digital, em especial os Objetos de Aprendizagem ${ }^{1}$ (OA), requerem um cronograma bem definido e organizado de atividades, com o intuito de otimizar o trabalho das equipes envolvidas neste processo [Monteiro et al. 2006].

Este trabalho apresenta através de Redes de Petri Coloridas (RPC) um modelo que permite especificar o fluxo de atividades e profissionais envolvidos na produção e validação dos OAs desenvolvidos pelo Le@ $\mathrm{d}$, bem como dimensionar as diversas equipes integrantes desse processo, calcular dinamicamente o tempo global para o desenvolvimento dos conteúdos didáticos de acordo com as dimensões e prazos de cada equipe, simular e calcular impactos causados por imprevistos no processo de produção, tais como a afastamento médico de membros das diversas equipes, e analisar falhas e gargalos na produção em geral.

Este documento é organizado da seguinte maneira: após a introdução realizada nesta seção, os trabalhos relacionados são apresentados na seção 2. A seção 3 traz uma introdução às Redes de Petri Coloridas. O Modelo de Produção de Objetos de Aprendizagem adotado no Le@d é apresentado na seção 4. Na seção 5 é apresentada uma análise e validação do modelo proposto. Por fim, as considerações finais são apresentadas na seção 6.

\section{Trabalhos Relacionados}

Os processos e modelos de produção, muitas vezes tidos como instrumentos de regulação, representam, na realidade, a experiência acumulada de anos de trabalho organizada por empresas e profissionais, que compilaram as melhores práticas para lidar mais próativamente com os riscos inerentes à execução dos mais variados serviços, incorporando mecanismos de controle que reduzem o nível de erros dos profissionais, levando-os de forma mais segura à finalização dos trabalhos [Castro and Barreto 2008].

As subseções seguintes apresentam as principais referências e seus modelos para produção de OAs.

\subsection{Modelo de Produção do RIVED}

O RIVED, Rede Interativa Virtual de Educação, é um programa da Secretaria de Educação a Distância (SEED) do MEC, cujo objetivo é a produção de OAs [RIVED 2014]. O modelo produtivo do RIVED é dividido em seis fases:

- Fase 01: Nesta fase, os professores conteudistas e designers instrucionais (DI) definem as abordagens técnicas e pedagógias do OA. Essa fase encerra-se com o General Designer (GD), um documento que descreve as atividades e objetivos do conteúdo educacional educacional.

- Fase 02: O GD é submetido a outras equipes de produção de OAs com o objetivo de receber críticas e sugestões sobre o processo descrito neste documento.

- Fase 03: Nesta fase, com base nos pareceres emitidos na fase anterior, são geradas as especificações de cada objeto.

\footnotetext{
${ }^{1}$ Um OA pode ser compreendido como qualquer entidade, digital ou não, que pode ser usada, reutilizada ou referenciada durante o processo de aprendizagem provido por algum tipo de tecnologia [IEEE LTSC 2010], úteis ao ensino ou aprendizagem.
} 
- Fase 04: Codificação do OA.

- Fase 05: Desenvolvimento de aplicação do conteúdo.

- Fase 06: Catalogação e publicação dos OAs na Web.

\subsection{Modelo de Produção do Sophia}

O projeto Sophia [Castro and Barreto 2008] visa auxiliar os alunos do curso de Tecnologia em Análise e Desenvolvimento de Sistemas (TADS), ofertado na modalidade a distância da Universidade do Vale do Itajaí (Univali), quanto à realização de atividades e com conteúdo de apoio às disciplinas.

O processo de produção adotado no Sophia é composto por três fases distintas:

- Projeto: Envolve a descrição detalhada da estrutura do OA. Atividades como definição de mídias e conteúdos, bem como o planejamento das atividades necessárias para seu desenvolvimento, alocação de recursos e definição do cronograma estão descritos nesta fase.

- Desenvolvimento: Contempla a produção do OA definido na etapa anterior, deixando-o pronto para disponibilizá-lo aos alunos. Nesta fase o OA passa por uma sub-etapa de revisão, realizada pelo professor conteudista.

- Distribuição: Esta etapa envolve a disponibilização e avaliação do material por tutores e alunos, visando a melhoria contínua do OA produzido.

\subsection{Modelo de Produção ADDIE}

Mustaro [Mustaro and Silveira 2007] sugere a utilização do modelo ADDIE como guia para o desenvolvimento de OAs. Este é composto por cinco etapas:

- Análise: Envolve a determinação dos objetivos, principalmente pedagógicos, do OA.

- Projeto: A fase de projeto acontece paralelamente à fase anterior. Compreende basicamente o plano de construção de cenários do OA. Também define estratégias, interatividade, feedback, usabilidade, entre outros.

- Desenvolvimento: Compreende a programação do OA. Nesta fase são produzidas as interfaces, adição de exercícios, dentre outros.

- Implementação: Para esta fase, faz-se necessário que o OA esteja disponível em um Ambiente Virtual de Aprendizagem (AVA). Neste estágio são catalogados os metadados e avaliados a integração entre o AVA e o OA.

- Avaliação: Esta é a última fase, que compreende todo o processo de desenvolvimento. Nela o objeto de aprendizagem é avaliado em questão do aprendizado que pode proporcionar. Essa avaliação deve ocorrer com o auxílio de alunos e professores.

Todos os trabalhos relacionados nesta seção apresentam uma preocupação na qualidade do OA produzido, incluindo sempre uma fase de avaliação e a melhoria do conteúdo produzido. O modelo adotado pelo Le@d formaliza a experiência de cinco anos na produção de OAs para o público com deficiência física e auditiva e, assim como os demais, também inclui uma fase de testes e evolução do material produzido. A especificação do fluxo de atividades por Redes de Petri Coloridas permite, dentre outras coisas, contabilizar dinamicamente os custos, dimensionar equipe e identificar entraves no processo de produção de conteúdos.

Na seção seguinte é apresentada uma introdução as Redes de Petri Coloridas. 


\section{Redes de Petri Coloridas}

Redes de Petri Coloridas (RPC) é uma linguagem gráfica para a construção de modelos de sistemas de eventos discretos e análise de suas propriedades. Estas combinam as capacidades das Redes de Petri (PN) com as capacidades de uma linguagem de programação de alto nível [Jensen and Kristensen 2009].

O tempo desempenha um papel significativo numa vasta gama de sistemas concorrentes. O correto funcionamento de alguns desses sistemas depende crucialmente do tempo em que certas atividades ocorrem. RPC incluem o conceito de tempo, que torna possível capturar o tempo para a realização de uma atividade no sistema. Isso significa que RPC podem ser aplicadas em análises de desempenho em sistemas simulados, permitindo investigar métricas diversas tais como tempo de produção de uma web aula ou tempo de validação de um objeto de aprendizagem, e para a modelagem e validação de sistemas em tempo real.

A estrutura principal de uma RPC consiste basicamente de três estruturas: lugares (representados por círculos ou elipses), transições (representados por retângulos) e arcos (representados por setas direcionais).

Lugares são utilizados para especificar um estado ou condição de um componente do sistema, enquanto que as transições representam eventos ou operações. Para interligar os componentes de uma RPC, existem dois tipos de arcos direcionados: arcos de entrada, que ligam uma transição a um lugar, e os arcos de saída, que ligam um lugar a uma transição.

A dinâmica da rede apoia-se no uso de fichas, que são depositadas em lugares. As fichas podem representar recursos ou condições, descrevendo o estado da rede em um instante no tempo. Estas são inseridas ou retiradas dos lugares através do disparo de uma transição. Para que uma transição possa ser disparada é necessário que haja uma quantidadede fichas nos lugares de entrada igual ou superior aos pesos dos seus respectivos arcos de entrada.

As expressões nos arcos determinam as fichas que serão removidas dos lugares de entrada e quais serão adicionadas aos lugares de saída quando da ocorrência de uma transição. Quando a expressão do arco avaliado é verdadeira a transição é habilitada e pode ocorrer (ser disparada), representando uma mudança de estado do sistema. Assim, após o disparo, fichas são inseridas nos lugares de saída da transição de acordo com o peso dos arcos de saída. É através dos disparos das transições que pode-se visualizar o fluxo de controle e de informações no sistema. Um estudo mais aprofundado sobre as RPC é fornecido por [Jensen and Kristensen 2009].

Para a construção do modelo foi utilizado o CPNTools ${ }^{2}$, uma ferramenta de modelagem, análise e simulação de RPC. A ferramenta foi desenvolvida na Universidade de Aarhus, Dinamarca, e é distribuída livremente para organizações não comerciais.

\section{O modelo de produção de Objetos de Aprendizagem do laboratório Le@d}

O modelo RPC de produção de OAs do Le@d é organizado em uma hierarquia de subredes (ou páginas). Uma subpágina é um modelo RPC, cuja semântica depende de outras páginas e/ou super-páginas [Jensen and Kristensen 2009].

\footnotetext{
${ }^{2}$ Www.cpntools.org
} 
A Figura 1 apresenta a página de maior nível hierárquico das redes que compõem o modelo de produção de OAs do Le@d. Nesse modelo os valores dos campos das fichas representam os artefatos para produção e equipes disponíveis para esta atividade.

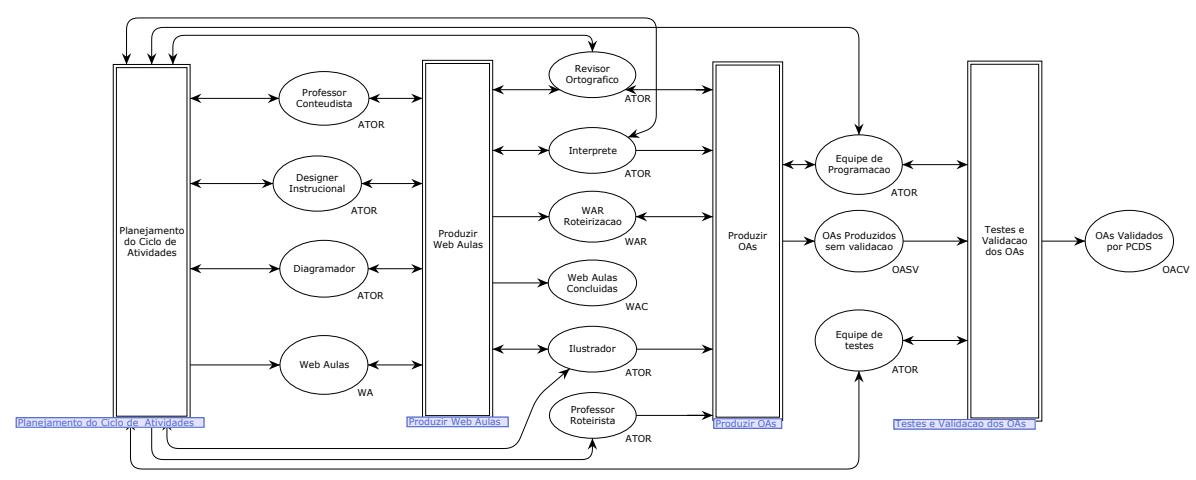

Figura 1. Modelo de produção de OAs em maior nível hierárquico

O Le@D opera em ciclos de atividades de doze meses. A primeira fase, denominada "Planejamento do Ciclo de Atividades" corresponde ao período em que a alta gerência do laboratório reune-se com representantes da empresa Dell Computadores para programar as atividades desenvolvidas durante o ciclo. Como resultado desta etapa temse o Documento de Escopo Projeto (DEP). Neste é possível verificar quantos conteúdos didáticos digitais (web aulas, OAs, video-aulas, dentre outros) serão produzidos e qual equipe foi dimensionada para tal atividade.

A fase seguinte, denominada "Produzir Web Aulas" é a etapa onde Professores Conteudistas (PC), especialistas no assunto, produzem aulas no formato web e que devem ser publicadas no EA2PCD, Ambiente Virtual de Aprendizagem (AVA) desenvolvido pelo laboratórioLe@D.

A efetiva produção dos OAs, realizados na etapa "Produzir OAs", tem como matéria-prima web aulas analisadas por um DI e revisadas gramaticalmente, e gera como resultado um OA para cada web aula produzida na etapa anterior. O fim dessa fase dá-se com a publicação do OA, ainda sem validação pelos PCDs no AVA do Le@D.

A quarta e última fase, denominada "Testes e Validacao dos OAs", compreende a aplicação do OA produzido à equipe de testes, compostas por testadores de software e PCDs. O resultado dessa fase gera um OA testado, validado, evoluído e aprovado por um grupo de pessoas que têm as mesmas deficiências do público alvo do material produzido.

Devido limitações de espaço, nas subseções seguintes apresentaremos apenas os detalhes das fases "Produzir Web Aulas" e "Produzir OAs". O modelo completo pode ser obtido no endereço http://interred.ifce.edu.br/sbie2014/ead.cpn.

\subsection{Produzir Web Aulas}

A Figura 2 apresenta a sub-rede modelada pela transição de substituição 3 "Produzir Web Aulas". Fichas do tipo ATOR representam os profissionais envolvidos em cada etapa do processo. O lugar "Professor Conteudista" modelam os professores responsáveis pela

\footnotetext{
${ }^{3}$ Representada por um retângulo de moldura dupla, uma transição de substituição indica que o processo a ela associado é modelado em uma sub-rede, portanto, representado independentemente.
} 
concepção e elaboração das web aulas. Fichas do tipo WA modelam as web aulas a serem produzidas. No exemplo da Figura 2, dois professores foram alocados para a produção de dez web aulas.

O disparo da transição "Produzir Web Aulas", que representa a atividade de produção de uma web aula, retira fichas do lugar "Web Aulas" e as deposita no lugar "Web Aulas Brutas". A função tpwebaula é define o tempo para realização desta atividade.

O lugar "Designer Instrucional" modela os Designers Instrucionais integrantes do processo de produção de conteúdos do Le@d, e a transição "DI Realizar Analise" representa as atividades realizadas por estes profissionais. O disparo da transição "DI Realizar Analise" retira fichas do lugar "Web Aulas Brutas" e as deposita no lugar "Web Aulas Analisadas". Esse movimento de fichas representa que uma web aula foi analisada por um DI, que fez adaptações pedagógicas sobre o assunto técnicos.

Após esta ação, as web aulas retornam ao PC, que decide por incorporar ou não as observações do DI ao material produzido. O tempo de atividade de um DI e o tempo de ajuste de um conteúdo por um PC são definidos através das funções tpanalise e ajustewa, respectivamente.

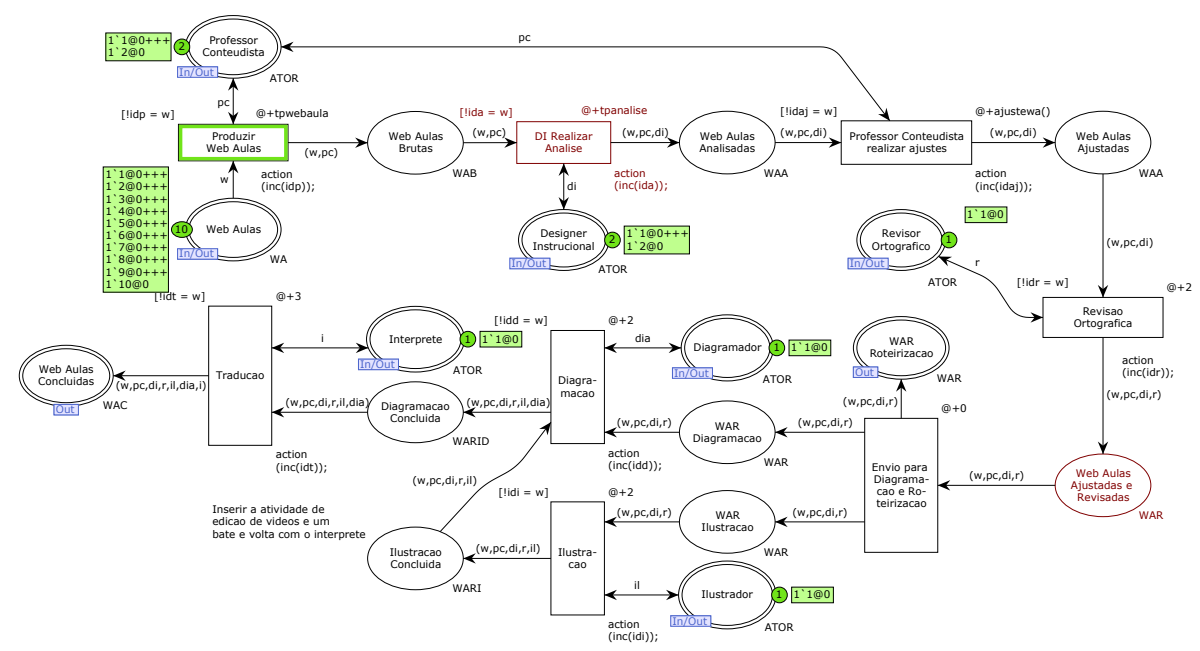

Figura 2. Sub-rede "Produzir Web Aulas - Detalhamento de atividades

Após o ajuste das web aulas é chegada a hora da revisão ortográfica. O lugar "Revisor Ortografico" modela os profissionais responsáveis por essa atividade. O disparo da transição "Revisão Ortográfica" representa a ação de revisão ortográfica de uma web aula. O tempo desta atividade no modelo da Figura 2 é fixo em duas Unidades de Tempo (UT), podendo ser alterado, caso necessário.

O lugar "Web Aulas Ajustadas e Revisadas" representa as aulas que já passaram pelo DI, PC e revisor ortográfico. O próximo passo é o envio desse conteúdo, ainda não concluído, para as equipes subsequentes. O disparo da transição "Envio para diagramação, ilustação, tradução e Roteirização" representa o envio deste material para as equipes de diagramação, ilustração, tradução e roteirização, simultaneamente.

Observa-se, segundo o modelo, que não é necessário que uma web aula esteja completamente concluída para começar a ser roteirizada. 
Os lugares "Diagramador", "Ilustrador" e "Interprete" representam os profissionais de diagramação, ilustração e tradução participantes deste processo. O disparo das transições "Diagramação", "Ilustração" e "Tradução" representam as atividades realizadas por estes profissionais. Note, segundo o modelo, que a diagramação é tarefa dependente da ilustração e tradução dos conteúdos. O resultado desse conjunto de atividades é obtida pelo disparo da transição "Postar Web Aulas", que representa a postagem das web aulas produzidas no EA2PCD.

\subsection{Produzir OAs}

A Figura 3 apresenta a sub-rede modelada pela transição de substituição "Produzir OAs". Fichas no lugar "Professor Roteirista" representam os professores responsáveis por criar o roteiro de construção dos OAs. Fichas no lugar "WAR Roteirização" representam as web aulas, ainda não finalizadas, que servem de matéria-prima para produção dos OAs. O disparo da transição "Produzir Roteiro OA" retira fichas do lugar "WAR Roteirização" e as deposita no lugar "Roteiro Concluido". Essa operação representa a atividade de produção de um roteiro de um OA. O tempo para esta atividade é representado pela função tproteiro.

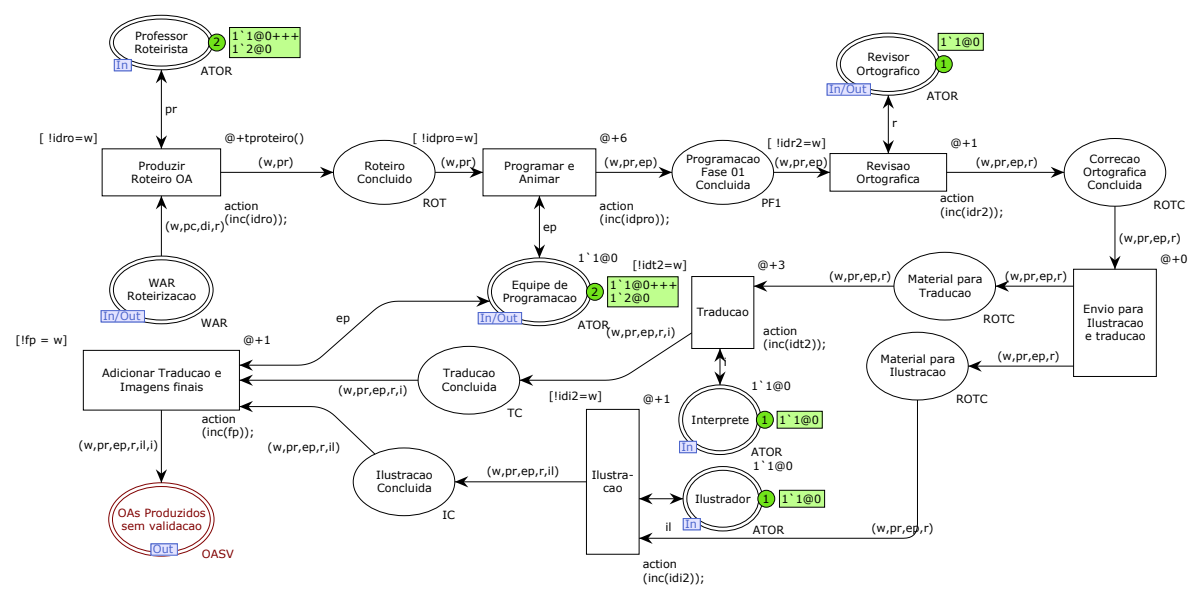

Figura 3. Sub-rede "Produzir OAs - Detalhamento de atividades

O disparo da transição "Programar e Animar" representa as atividades realizadas pela equipe de programação e animação do OA. O tempo de realização desta atividade segundo o modelo da Figura 3 é seis UT, mas assim como as outras atividades que têm tempo fixo, essa valor pode ser alterado para representar o acrescimo ou decrescimo de tempo para realização desta atividade. O fim de uma atividade de programação e animação é representada pelo deposito de fichas do tipo PF1 no lugar "Programacao Fase 01 Concluida".

Após a primeira etapa de programação, o OA ainda em estado "bruto" deve passar por revisões ortográficas. Assim como na sub-rede "Produzir Web Aulas", os corretores ortográficos são modelados por fichas no lugar "Corretor Ortográfico"e a ação realizada por este profissional é modelada pela transição "Revisão Ortográfica". Na sub-rede em discussão, o tempo médio para a revisão ortográfica de um OA é uma UT.

O resultado da revisão ortográfica, representado pelo depósito de fichas no lugar "Correcao Ortografica Concluida" deve ser enviado simultaneamente para o ilustrador e 
intérprete. O depósito de fichas nos lugares "Material para Ilustração" e "Material para Tradução" representam que os profissionais Ilustradores e Intérpretes têm insumos para realização de suas atividades.

O disparo das transições "Traducao" e "Ilustracao" representam as atividades realizadas por intérpretes e ilustradores, respectivamente, no processo de produção de um OA. Os tempo de cada atividade estão fixos em três e uma UT, conforme observado na Figura 3.

O final desta etapa caracteriza-se pelo depósito de fichas do tipo OASV no lugar "OAs produzidos sem validação". Estas representam que o OA foi concluído, entretanto ainda não passou pela validação da equipe de testes.

\section{Análise e Validação do Modelo}

As técnicas de análise e validação para RPCs são, em geral, adaptações de técnicas utilizadas para RPs. Desta forma, para análise e validação de uma RPC, pode-se empregar simulação, grafo de ocorrência e cálculo de invariantes [Jensen and Kristensen 2009].

A simulação consiste em executar a rede, interativa e automaticamente, e avaliar o comportamento do sistema modelado, possibilitando visualizar o resultado de uma sequência de eventos [Jensen and Kristensen 2009].

A fim de avaliar o tempo de produção de OAs levando-se em consideração o número de profissionais que compõem as diversas equipes envolvidas neste processo, dois cenários de simulação foram propostos: o primeiro deles assume que a produção ocorre dentro do esperado, sem imprevistos ou atrasos indevidos; O segundo simula atrasos por parte da equipe de DIs. Em ambos cenários, um DI é adicionado à equipe e os resultados desse incremento são avaliados.

As subseções seguintes apresentam detalhadamente os cenários de simulação propostos e realizam uma discussão sobre os resultados obtidos.

\subsection{Cenário de simulação 1}

O cenário inicial simula a produção de dez web aulas e dez OAs. As equipes de maior interesse têm seus tempos de atividade descritos através da Tabela 5.1. O fluxo de produção de conteúdo segue o modelo apresentado através da Figura 1. As equipes de revisores ortográficos, diagramação, ilustração, intérpretes, e testadores têm seus tempos de atividade fixos em duas, duas, duas, uma e sete UT, respectivamente.

Após a definição do cenário inicial, através de simulações, recuperamos o tempo global para produção de cada uma das web aulas e OAs. Após a recuperação desta informação, um DI é adicionado à equipe e os resultados desse incremento são avaliados.

\begin{tabular}{lcc}
\hline Equipe de Profissionais & Profissionais por Equipe & Tempo de uma atividade \\
\hline Professor Conteudista & 2 & 10 \\
\hline Designer Instrucional & 1 & 5 \\
\hline Professor Roteirista & 1 & 7 \\
\hline Programadores & 1 & 7 \\
\hline
\end{tabular}

Tabela 1. Cenário 1: Descritivo de Profissionais e Tempo de Atividades 


\subsubsection{Resultados Obtidos}

O gráfico da Figura 4 (A) representa os tempos de conclusão das web aulas e OAs de cada aula com a equipe descrita na Tabela 5.1.
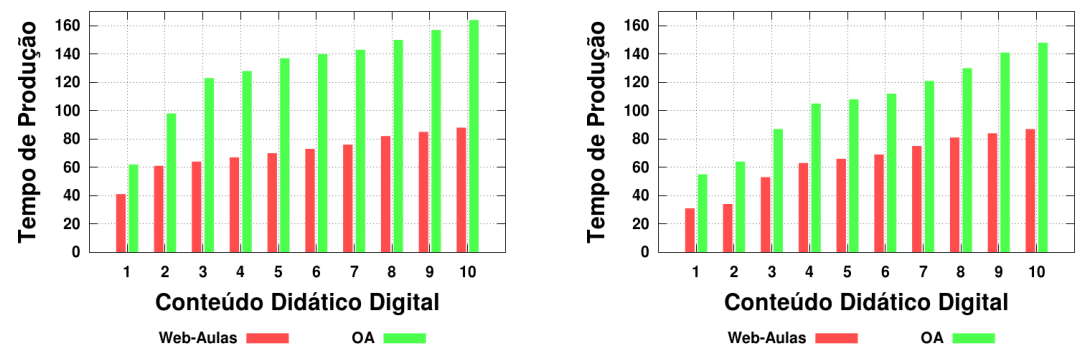

Figura 4. Tempo de produção dos artefatos programados (A) com um DI e (B) dois Dls.

Adicionado um DI ao processo e realizada nova simulação, obtêm-se os resultados exibidos através do gráfico da Figura 4 (B). Observa-se que a inclusão deste profissional diminui os tempos de produção dos artefatos, entretanto esse decréscimo de tempo não justifica a contratação de um novo profissional. Isso é devido a um DI ser suficiente para dar vazão ao trabalho de dois professores conteudistas. A permanência desse profissional na equipe mantém os dois DIs ociosos em cinquenta por cento do tempo.

\subsection{Cenário de simulação 2}

O segundo cenário é semelhante ao anterior, entretanto, o tempo de atividade de um DI varia entre sete e nove UT. Esse incremento de tempo pode significar um problema ocorrido com o profissional, como por exemplo uma doença ou dificuldade de locomoção devido a uma greve no serviço público de transporte. O restante dos profissionais e tempos de realização de suas atividades são iguais ao apresentados anteriormente.

\subsection{Resultados Obtidos}

A Figura 5 (A) apresenta os tempos de produção de web aulas e OAs para o cenário 5.2. Adicionando-se um DI, obtêm-se os resultados apresentados na Figura 5 (B).
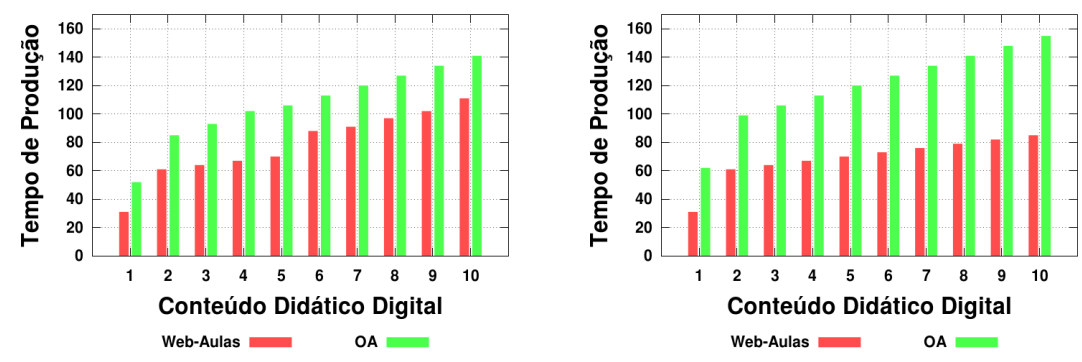

Figura 5. Tempo de produção dos artefatos programados (A) com um DI e (B) dois Dls.

Neste novo cenário de testes, percebe-se que a inclusão de um novo DI no processo diminui consideravelmente o tempo de produção de web aulas. Entretanto, o novo membro da equipe pouco influencia no tempo de produção dos OAs. Isso é devido à presença de um ou mais gargalos na equipe de produção dos objetos de aprendizagem. 


\section{Conclusões}

Este trabalho apresenta um modelo que permite especificar o fluxo de atividades e profissionais envolvidos na produção e validação dos OAs desenvolvidos pelo Le@ @, bem como dimensionar as diversas equipes integrantes desse processo, calcular dinamicamente o tempo global para o desenvolvimento dos conteúdos didáticos, simular e calcular impactos causados por imprevistos no processo de produção e analisar falhas e gargalos na produção em geral. Através do modelo é possível a maior alocação de atividades por equipe sem comprometer o prazo final de entrega dos artefatos.

O modelo é composto por quatro fases: Planejamento do Ciclo de Atividades, Produzir Web Aulas, Produzir OAs e, por fim, Testes e Validação dos OAs. Entretanto, devido às limitações de espaço, detalhamos apenas as duas fases centrais.

Dois cenários de simulação foram propostos a fim de avaliar o impacto do incremento de profissionais em diversas condições de produção de conteúdos: o primeiro deles avalia o incremento de um DI, que leva exatamente cinco UT para realização de uma atividade, enquanto dois PCs constroem aulas simultâneamente. O outro cenário simula imprevistos com o profissional DI durante o exercício de suas atividades.

Os resultados sugerem que o incremento de profissionais em equipe pode não trazer resultados que justifiquem sua incorporação ao processo produtivo. Em outras situações onde a adição de um profissional incrementa apenas parte de processo de produção de conteúdos, sugere-se que existam outras equipes que se encontram em seu limite máximo de atividades.

\section{Referências}

Castro, M. P. and Barreto, F. V. B. (2008). Proposta de um processo para produção de objetos de aprendizagem. Hífem. Uruguaiana.

IBGE (2014). Online: disponível em http://7a12.ibge.gov.br/vamos-conhecer-obrasil/nosso-povo/caracteristicas-da-populacao. Acesso em Maio de 2014.

IEEE LTSC (2010). Ieee learning technology steards committee. Online: disponível em http://ieeeltsc.org. Acesso em Dezembro de 2013.

Jensen, K. and Kristensen, L. M. (2009). Coloured Petri Nets: Modelling and Validation of Concurrent Systems. Springer.

Monteiro, B. S., Oliveira, H. P. C., Andrade, M. J. P., Golveia, T. B., Tavares, R., and Cabral, L. A. F. (2006). Metodologia de desenvolvimento de objetos de aprendizagem com foco na aprendizagem significativa. Simpósio Brasileiro de Informática na Educação. Distrito Federal.

Mustaro, P. N. and Silveira, I. F. a Omar, N. e. S. S. M. D. (2007). Structure of storyboard for interactive learning objects development. Informing Science Press, pages 253 279.

RIVED (2014). Rede internacional virtual de educação. Online: disponível em http://www.rived.mec.gov.br. Acesso em Janeiro de 2014. 